\title{
BMJ
}

\section{Effect of food intake during labour on obstetric outcome: randomised controlled trial}

\author{
Geraldine O'Sullivan, consultant anaesthetist, ${ }^{1}$ Bing Liu, research associate, ${ }^{2}$ Darren Hart, agenda for change \\ midwife, ${ }^{3}$ Paul Seed, senior lecturer in medical statistics, ${ }^{2}$ Andrew Shennan, professor of obstetrics ${ }^{2}$
}

'Department of Anaesthesia, St Thomas' Hospital, Guy's and St Thomas' NHS Foundation Trust, London SE1 7EH

${ }^{2}$ Maternal and Fetal Research Unit, King's College London, London SE1 7EH

3Guy's and St Thomas' NHS

Foundation Trust

Correspondence to: A Shennan andrew.shennan@kcl.ac.uk

Cite this as: BMJ 2009;338:b784 doi:10.1136/bmi.b784

\section{ABSTRACT}

Objective To investigate the effect of feeding during labour on obstetric and neonatal outcomes.

Design Prospective randomised controlled trial.

Setting Birth centre in London teaching hospital.

Participants 2426 nulliparous, non-diabetic women at term, with a singleton cephalic presenting fetus and in labour with a cervical dilatation of less than $6 \mathrm{~cm}$. Intervention Consumption of a light diet or water during labour.

Main outcome measures The primary outcome measure was spontaneous vaginal delivery rate. Other outcomes measured included duration of labour, need for augmentation of labour, instrumental and caesarean delivery rates, incidence of vomiting, and neonatal outcome.

Results The spontaneous vaginal delivery rate was the same in both groups (44\%; relative risk 0.99, $95 \%$ confidence interval 0.90 to 1.08 ). No clinically important differences were found in the duration of labour (geometric mean: eating, 597 min $v$ water, $612 \mathrm{~min}$; ratio of geometric means $0.98,95 \%$ confidence interval 0.93 to 1.03 ), the caesarean delivery rate $(30 \% \mathrm{v} 30 \%$; relative risk $0.99,0.87$ to 1.12 ), or the incidence of vomiting ( $35 \% v 34 \%$; relative risk $1.05,0.9$ to 1.2). Neonatal outcomes were also similar. Conclusions Consumption of a light diet during labour did not influence obstetric or neonatal outcomes in participants, nor did it increase the incidence of vomiting. Women who are allowed to eat in labour have similar lengths of labour and operative delivery rates to those allowed water only.

Trial registration Current Controlled Trials ISRCTN33298015.

\section{INTRODUCTION}

The obstetric guideline of the American Society of Anaesthesiologists, published in 2007, states that "the oral intake of solids during labour increases maternal complications," and that "solid foods should be avoided in labouring patients." It also recommends that "the oral intake of modest amounts of clear liquids (e.g. water, clear tea, black coffee, and sports drink) may be allowed for uncomplicated labouring patients." The policy of fasting during labour was adopted after Mendelson published his now classic description of acid pulmonary aspiration in $1946 .{ }^{2} \mathrm{Pul}-$ monary aspiration in obstetrics has declined dramatically in recent years, probably as a result of the increased use of regional anaesthesia for operative deliveries, the perioperative use of $\mathrm{H}_{2}$ antagonists and proton pump inhibitors, and widespread improvements in the training of obstetric anaesthetists. ${ }^{3-8}$ Consequently, in some countries, the policy of routine fasting for all parturients has been challenged. ${ }^{9-12}$ Relaxation of the rule has been more prevalent in Europe than in the United States. In the Netherlands 79\% of clinicians allow food intake in labour, ${ }^{11}$ and among 351 birth units in the United Kingdom 32\% allowed food and drink. ${ }^{9}$ Although it has not been formally evaluated, many clinicians have observed that women request food in labour. In contrast, in the United States oral intake during labour is limited primarily to clear liquids.

To date five randomised controlled trials, in fewer than 1000 women, have evaluated the influence of calorific intake on outcome of labour. ${ }^{13-17}$ Four studies reported no significant differences in either the mode

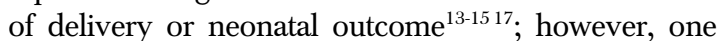
study reported longer labours in women who consumed food, ${ }^{13}$ and another showed an almost threefold increase in the rate of caesarean delivery. ${ }^{16}$ These, however, had limited power to detect clinically important differences or show the clinical equivalence of treatments.

The current rationale for women fasting during labour is to protect them from pulmonary aspiration should general anaesthesia be needed for an emergency operative delivery. However, prolonged fasting in labour has never been proved to influence the incidence of pulmonary aspiration, and some clinicians and midwives consider that preventing food intake can be detrimental to the mother, her baby, and the progress of labour. ${ }^{18-22}$ Whether food intake in labour will influence the ability to deliver normally, the length of labour, or other obstetric and neonatal end points is not known.

We did a randomised trial to determine the influence of consumption of food during labour on the rate of spontaneous vaginal delivery. Other outcomes measured included duration of labour, incidence of vomiting, and neonatal outcome. 


\section{METHODS}

We did the trial at Guy's and St Thomas' Hospital in London between June 2001 and April 2006.

\section{Selection of patients}

Women were informed of the study during the second or third trimesters of pregnancy. This was to ensure previous knowledge and understanding of the aims of the study before women gave written consent later in the delivery suite. We invited women to participate if they were over 18 years of age, were nulliparous, were more than 36 weeks' gestation, did not have diabetes, had a singleton cephalic presenting fetus, and were in labour with a cervical dilatation of less than $6 \mathrm{~cm}$. We included women having induction or augmentation of labour. We did not recruit multiparous women, given their potentially quicker labours and low operative delivery rates - that is, less exposure to the intervention and low prevalence of the primary outcome measure.

We excluded women who had a known obstetric or medical complication that could have increased the likelihood of an operative delivery, were in severe pain, intended to use parenteral opioids for analgesia during labour, or were unable to understand English (and no interpreter was available).

\section{Study design}

After women gave informed, written consent, the attending midwife randomised them either into the "eating" or the "water only" group. Entry of a woman's initials, hospital number, and date of birth on to a dedicated computer on the labour ward automatically generated the allocation group together with a study number, which was then recorded on the outcome sheet. These data could, if necessary, be verified against the computer randomisation at a later time.

\section{Dietary advice}

After randomisation, women in the eating group were advised to consume a low fat, low residue diet at will during their labour. The emphasis was on small regular amounts of food rather than eating set regular meals. Suggested foods included bread, biscuits, vegetables, fruits, low fat yoghurt, soup, isotonic drinks, and fruit juice. All women had free access to water. Women in the water only group were advised to have ice chips and water only. Women were told that eating was not recommended in labour (as was the policy in the hospital at the time), but they were actively encouraged to do so if randomised to the feeding arm. Women were made aware that this is increasingly practised in many units. Women in the water only group were encouraged not to eat if they requested to do so. Light food was made available on the labour ward, or women could bring in their own food.

\section{Data collection}

The attending midwife was responsible for entering outcome data on to a data sheet. Age, ethnicity, food intake for six hours before labour and during labour, the incidence of vomiting (once and more than once), and the indication for intravenous fluids were recorded. Obstetric outcomes included the mode of delivery (primary outcome), the duration of labour, and use of intravenous oxytocin for the augmentation of labour. We categorised food intake in the six hours before labour as no intake, snacks, a light meal, or a large meal. We categorised food intake during labour as no intake, water only, or calorific drinks/solids. The exact food consumed was at each woman's discretion. The trial coordinator was responsible for training midwives on the study protocol and adherence to the protocol and for the daily collection of data sheets. All data were then entered on to a Microsoft Excel 2000 spreadsheet.

\section{Obstetric management}

The attending obstetricians and midwives made all the relevant decisions about the woman's obstetric management but obviously could not be blinded to trial allocation. The people deciding on obstetric interventions were generally unaware of the trial intervention allocation and had no vested interest in the study. Vaginal dilatation was assessed at four hourly intervals. Continuous external fetal heart rate monitoring and tocodynamometry were used as indicated. Oxytocin infusion, when indicated, was administered according to the hospital protocol. No routine antacid was administered. The decision to proceed to an operative delivery was made by the duty obstetrician. The attending midwife recorded the Apgar scores at delivery.

\section{Analgesia during labour}

The women had access to a birthing pool for both labour and delivery. They could also choose 50:50 nitrous oxide and oxygen (Entonox) or epidural analgesia. A low dose local anaesthetic and opioid solution (bupivacaine $0.1 \%$ with fentanyl $2 \mu \mathrm{g} / \mathrm{ml}$ ) was used for epidural analgesia. Analgesia was initially achieved with 10-15 $\mathrm{ml}$ of this solution and maintained with the same solution through a patient controlled epidural analgesia system. This system was maintained by using a bolus dose of $9.9 \mathrm{ml}$ with a lockout time of 20 minutes. The maximum dose of the local anaesthetic and opioid solution was $30 \mathrm{ml} / \mathrm{hour}$.

\section{Outcome measures}

The primary outcome was the rate of spontaneous vaginal delivery, and the secondary outcome was the duration of labour. We defined duration of labour as the time from the onset of the active phase of first stage until the end of the second stage of labour, when the baby was delivered. For women who presented on the labour ward after the establishment of active labour, the duration was recorded as the time from admission until the end of the second stage of labour. We considered the duration of labour to be zero in those women who had a caesarean delivery before the establishment of the active phase of labour. The instrumental delivery rate, the caesarean delivery rate, and the need for augmentation of labour were also recorded.

Neonatal outcome-One minute and five minute Apgar scores were recorded, as was the fetal weight. 


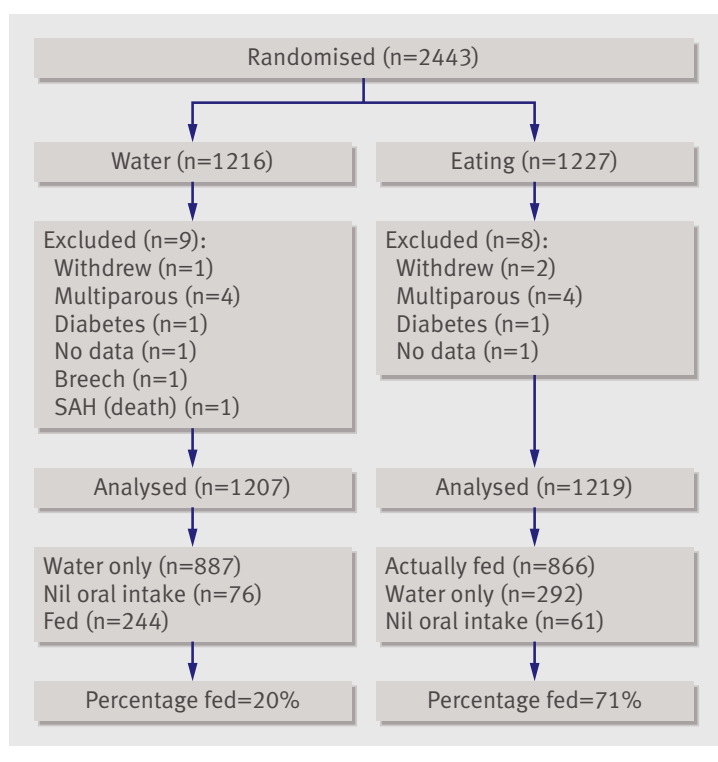

Flow of participants through study. PET=pre-eclamptic toxaemia; $\mathrm{SAH}=$ subarachnoid haemorrhage

The need for admission to the neonatal intensive care unit or special care baby unit was also assessed.

\section{Statistical analysis}

We determined that 1126 women would give 90\% power to detect a clinically important difference in the spontaneous vaginal delivery rate of $6.7 \%$ (60\% v66.7\%; risk ratio 1.11). The clinicians involved in the trial, after discussion of the relevant issues, agreed that evidence of a difference of $6.7 \%$ would be clinically important, having the potential to affect clinical practice. Assuming that duration of labour is normally distributed and using a mean duration from a similar but unpublished study of 297 women, ${ }^{13}$ we calculated that 1212 participants would be needed for a $10 \%$ change in the duration of labour. In setting the clinically important differences,

Table 1|Baseline characteristics. Values are numbers (percentages) unless stated otherwise

\begin{tabular}{lcc} 
Characteristic & Eating $(\mathrm{n}=1219)$ & Water $(\mathrm{n}=1207)$ \\
Age: & & $29(6)$ \\
\hline Mean (SD) & $18-44$ & $18-47$ \\
\hline Range & $751(62)$ & $741(61)$ \\
\hline Ethnic group: & $285(23)$ & $281(23)$ \\
\hline White & $183(15)$ & $185(15)$ \\
\hline African or Caribbean & $79(6)$ & $61(5)$ \\
\hline Other & $438(36)$ & $434(36)$ \\
\hline Pre-labour food intake: & $441(36)$ & $395(33)$ \\
\hline Large meal & $261(21)$ & $317(26)$ \\
\hline Light meal & & $92(8)$ \\
\hline Snack & $117(10)$ & $233(19)$ \\
\hline No food & $212(17)$ & $813(67)$ \\
\hline Labour induction: & $804(66)$ & $3428(520)$ \\
\hline Prostaglandin only & $3421(472)$ & \\
\hline Prostaglandin plus oxytocin & & \\
\hline Epidural analgesia & & \\
\hline Mean (SD) baby's birth weight (g) & & \\
\hline
\end{tabular}

we made allowance for a possible substantial non-compliance rate. We did the analysis according to the intention to treat principle, making comparisons between groups as randomised. Results are presented as estimates with $95 \%$ confidence intervals, to facilitate determination of clinical equivalence. ${ }^{23} \mathrm{We}$ analysed data by using Excel 2000, Student's $t$ test, and Stata version 9. As we found that duration of labour had a strongly skewed distribution, we used log transformations, and results are reported as geometric means and as ratios of geometric means.

\section{RESULTS}

We randomised 2443 nulliparous women to this trial, of whom 17 were excluded from the final analysis. The figure shows the reasons for exclusion. Of the 2426 participants included in the analysis, 1219 were in the eating group and 1207 were in the water only group. In view of the round the clock recruitment, we were unable to ensure accurate data on the number of women approached and their reasons for declining. Women were not approached when midwives were untrained in the study protocol. Women generally refused if they definitely wanted to eat. During the study period 12918 nulliparous women laboured with singleton babies with cephalic presentation, although not all these women were eligible if in advanced labour ( $>5 \mathrm{~cm}$ dilatation). The two randomised groups were comparable with respect to age, ethnic group, pre-labour food intake, need for intravenous fluids, and use of prostaglandin and oxytocin. The birth weights of the neonates were similar in both groups (table 1). One serious adverse event (maternal death) occurred in the water only group, and this was due to a subarachnoid haemorrhage. No cases of pulmonary aspiration of gastric contents occurred.

One hundred and thirty seven women $(5.6 \%$ of participants) consumed nothing during labour. Of the women allocated to water only, $20 \%$ failed to adhere to the protocol and consumed food. Of those allocated to the eating group, $29 \%$ chose not to eat. Food consumed by the women included fruit juice, soup, cereal, biscuits, fruits, chocolate, toast, vegetable stew, Danish pastry, sandwiches, burgers, chicken, and rice.

\section{Primary outcome}

By intention to treat, we found no significant difference in the rate of normal vaginal delivery between the two groups. The rates were 533/1219 (44\%) in the eating group and 534/1207 (44\%) in the water only group (risk ratio $0.99,95 \%$ confidence interval 0.90 to 1.09 ) (table 2).

\section{Secondary and neonatal outcomes}

We found no significant difference in the duration of labour between the two groups (table 2). The geometric mean duration of labour was 597 minutes for the eating group and 612 minutes for the water group (ratio of geometric means $0.975,95 \%$ confidence interval 0.927 to 1.025$)$. 
Table 2 | Primary and pre-defined secondary maternal outcomes. Values are numbers (percentages) unless stated otherwise

\begin{tabular}{|c|c|c|c|c|}
\hline Outcome & $\begin{array}{c}\text { Eating } \\
(n=1219)\end{array}$ & $\begin{array}{c}\text { Water } \\
(n=1207\end{array}$ & $P$ value & $\begin{array}{c}\text { Comparison } \\
(95 \% \mathrm{Cl})\end{array}$ \\
\hline Normal vaginal delivery* & $533(44)$ & $534(44)$ & 0.77 & $0.99 \dagger(0.91$ to 1.09$)$ \\
\hline Instrumental delivery & $324(27)$ & $310(26)$ & 0.64 & $1.04 \dagger(0.91$ to 1.19$)$ \\
\hline Caesarean section & $362(30)$ & $363(30)$ & 0.86 & $0.987 \dagger(0.87$ to 1.12$)$ \\
\hline Vomited & $430(35)$ & $406(34)$ & 0.41 & $1.05 \dagger(0.94$ to 1.17$)$ \\
\hline Oxytocin for augmentation & $647(53)$ & $673(56)$ & 0.19 & $0.95 \dagger(0.88$ to 1.02$)$ \\
\hline Intravenous fluid $>500 \mathrm{ml}$ & $820(67)$ & $838(69)$ & 0.25 & $0.969+(0.92$ to 1.02$)$ \\
\hline \multicolumn{5}{|l|}{ Length of labour (min): } \\
\hline Geometric mean & 597 & 612 & - & \multirow{2}{*}{$0.975 \ddagger(0.927$ to 1.025$)$} \\
\hline Median (interquartile range) & $669(437-929)$ & $658(432-905)$ & - & \\
\hline
\end{tabular}

*Primary outcome.

†Risk ratio.

‡Ratio of geometric means.

We found no significant difference between the groups with respect to the rate of instrumental vaginal delivery or caesarean delivery, the incidence of maternal vomiting, or the use of epidural analgesia or of oxytocin for augmentation of labour (table 2). Although ketonuria was a pre-defined end point in this study, a large proportion of women did not have urinalysis in late labour, and we therefore had to abandon this as a meaningful end point.

When we compared women who actually fed with those who did not the results were similar (normal vaginal delivery $44 \% v 44 \%$; risk ratio $0.99,0.91$ to 0.108 ). No cases of aspiration occurred during the study period.

We found no differences between the two groups with respect to Apgar scores or admission to neonatal intensive care or special care units (table 3). The confidence intervals were sufficiently tight to exclude clinically important differences.

\section{DISCUSSION}

This study, the largest prospective randomised study to have evaluated the influence of food consumption during labour, suggests that this practice does not influence obstetric or neonatal outcomes. Feeding does not shorten labour, nor does it increase the chance of a normal delivery; withholding food is therefore not detrimental to these end points. We are confident that no clinically important difference existed between the two treatment arms in either the mode of delivery or duration of labour, as shown by the confidence intervals.

Considering previous data, ${ }^{24}$ one study of 297 women found a significantly longer duration of labour in women who consumed food during labour, ${ }^{13}$ whereas Scheepers et al showed a significantly increased

Table 3|Pre-defined secondary neonatal outcomes. Values are numbers (percentages) unless stated otherwise

\begin{tabular}{|c|c|c|c|c|}
\hline Outcome & Eating $(n=1219)$ & Water only $(n=1207)$ & $P$ value & Risk ratio $(95 \% \mathrm{Cl})$ \\
\hline Apgar score $5 \min \leq 7$ & $16(1.3)$ & $22(1.8)$ & 0.33 & 0.72 (0.38 to 1.36$)$ \\
\hline Apgar score $5 \min \leq 4$ & $4(0.33)$ & $9(0.75)$ & 0.18 & 0.44 (0.14 to 1.42$)$ \\
\hline Admission to SCBU/ICU & $61(5.0)$ & $62(5.2)$ & 0.81 & $0.96(0.68$ to 1.35$)$ \\
\hline
\end{tabular}

caesarean delivery rate (risk ratio $2.9,1.29$ to 6.54 ).$^{16}$ In the light of this, much larger study, both of these may be incorrect. A later study by Scheepers et al investigated the impact of intake of carbohydrate solution just before the start of the second stage of labour on maternal and fetal metabolism and clinical outcome and found no differences in the rates of instrumental and caesarean delivery between the groups. ${ }^{25}$ The other three trials that have evaluated calorific intake during labour showed no significant effect on labour or neonatal outcome but were not sufficiently powered to evaluate these outcomes. ${ }^{141517}$ Scrutton et al showed that eating in labour prevented the development of ketosis but significantly increased residual gastric volume,${ }^{14}$ whereas Kubli et al showed that isotonic drinks reduced maternal ketosis during labour without increasing gastric volume. ${ }^{15}$ In accordance with our findings, Tranmer et al reported no difference in the incidence of dystocia, the need for augmentation of labour, the duration of labour, or the method of delivery between the intervention (unrestricted oral carbohydrate intake) and control (ice chips and water) groups. ${ }^{17}$

Parenteral opioid analgesia was an exclusion factor in our study, as opioids cause a significant delay in gastric emptying during labour and may increase the risk of vomiting. ${ }^{26}$ The use of low dose local anaesthetic and opioid solutions for epidural analgesia was not an exclusion criterion. Low dose local anaesthetic and opioid solutions containing fentanyl do not significantly prolong gastric emptying, ${ }^{2728}$ unless the amount of fentanyl administered exceeds $100 \mu \mathrm{g}$.

The main concern of anaesthetists with regard to eating during labour is the potential risk of pulmonary aspiration, should general anaesthesia be needed. The triennial reports of the UK Confidential Enquiries into Maternal and Child Health indicate that the incidence of pulmonary aspiration of gastric content has declined considerably in the past 20 years, despite an increasingly liberal attitude to eating during labour..$^{3-8}$ In the most recent report (2003-5), six direct anaesthesia related maternal deaths occurred among 2113831 deliveries, none of which was associated with pulmonary aspiration. This is consistent with the finding of the review of 133 aspirations during anaesthesia from the Australian Anaesthesia Incident Monitoring Study. ${ }^{29}$

Equally, denial of food can be seen as authoritarian and intimidating, which may for some women increase feelings of fear and apprehension during labour. ${ }^{30}$ Eating and drinking may allow mothers to feel normal and healthy. ${ }^{31}$ Women tend to follow their normal dietary pattern in early labour but reduce their food intake as labour becomes more painful. ${ }^{1732}$ In Mendelson's original paper, mortality was related only to choking on solid food. In the light of this and the absence of evidence to the contrary, women who wish to eat should be discouraged from solid food. However, given the current extremely low incidence of pulmonary aspiration in the obstetric population, some clinicians may decide, in low risk labouring women, that the consumption of a light diet could be left to the woman's discretion. 


\section{WHAT IS ALREADY KNOWN ON THIS TOPIC}

The practice of fasting women during labour was intended to protect them from pulmonary aspiration should general anaesthesia be needed for an emergency operative delivery

Prolonged fasting in labour has never been proved to influence the incidence of pulmonary aspiration

Some clinicians and midwives argue that preventing food intake during labour can be detrimental to the mother, her baby, and the progress of labour

\section{WHAT THIS STUDY ADDS}

Eating did not influence obstetric (mode of delivery or duration of labour) or neonatal outcomes

No evidence of harm was found, but the power was insufficient to imply safety owing to the current extremely low incidence of acid pulmonary aspiration in obstetrics

If low risk women are offered a light, easily digestible diet during labour they should be advised that this will not improve their obstetric and neonatal outcome

\section{Conclusions}

This randomised controlled trial was designed to evaluate whether the policy of fasting during labour is justified in modern obstetric practice. We found that eating during labour did not influence neonatal or obstetric outcomes, including the rates of spontaneous and operative delivery and the duration of labour. This trial was unable to show evidence of harm as it was not powered to do so given the low prevalence of aspiration, and this question of harm is unlikely to be answered in clinical trials because of the extremely low incidence of acid pulmonary aspiration. The biggest evidence for safety is probably related to the lack of aspiration related morbidity in recent years, ${ }^{7}$ in spite of an increasing trend to feeding in labour over the past decades. ${ }^{910}$ Aspiration pneumonitis/pneumonia is significantly associated with intubation and ventilation. ${ }^{33}$ In modern obstetric practice it is the use of regional anaesthesia, thereby avoiding intubation, rather then fasting regimens that is likely to have reduced mortality from aspiration. Although the National Institute for Health and Clinical Excellence has recommended, on the basis of consensus opinion, that women in normal labour may eat/drink in labour, our trial shows that this will not improve their obstetric and neonatal outcomes.

We are indebted to the mothers, midwives, anaesthetists, and obstetricians at St Thomas' Hospital for their cooperation and consistent support. Contributors: GO'S and AS successfully applied for the funding for this project. $\mathrm{BL}$ and $\mathrm{DH}$ ran the trial. $\mathrm{BL}$ and $\mathrm{PS}$ analysed the data. $\mathrm{AS}$ is the guarantor

Funding: This study was supported by a grant from the Obstetric Anaesthetists' Association and the Special Trustees of the St Thomas' Hospital. AS is supported by Tommy's-The Baby Charity. Competing interests: None declared.

Ethical approval: The research protocol was reviewed and approved by the Local Ethics Committee.

1 American Society of Anesthesiologist's Task Force on Obstetric Anesthesia. Practice guidelines for obstetric anesthesia. Anesthesiology 2007;106:843-63.

2 Mendelson CL. The aspiration of stomach contents into the lungs during obstetric anesthesia. Am J Obstet Gynecol 1946;52:191-206.

3 Department of Health, Welsh Office, Scottish Home and Health Department, and Department of Health and Social Services, Northern Ireland. Report on confidential enquiries into maternal deaths in the United Kingdom 1988-1990. London: HMSO, 1994.

4 Department of Health, Welsh Office, Scottish Home and Health Department, and Department of Health and Social Services, Northern
Ireland. Report on confidential enquiries into maternal deaths in the United Kingdom 1991-1993. London: HMSO, 1996.

5 Department of Health, Welsh Office, Scottish Home and Health Department, and Department of Health and Social Services, Northern Ireland. Report on confidential enquiries into maternal deaths in the United Kingdom 1994-1996. London: Stationery Office, 1998.

6 Department of Health, Scottish Executive Health Department, and Department of Health, Social Services and Public Safety, Northern Ireland. Why mothers die: fifth report on confidential enquiries into maternal deaths in the United Kingdom, 1997-1999. London: RC OG Press, 2001

7 Department of Health. Why mothers die 2000-2002: report on confidential enquiries into maternal deaths in the United Kingdom. London: CEMACH Publication, 2004.

8 Lewis G, ed. Saving mothers' lives: reviewing maternal deaths to make motherhood safer-2003-2005. The seventh report on confidential enquiries into maternal deaths in the United Kingdom. London: CEMACH, 2007.

9 Michael S, Reilly CS, Caunt JA. Policies for oral intake during labour: a survey of maternity units in England and Wales. Anaesthesia 1991;46:1071-3.

10 Hawkins J, Gibbs C, Martin-Salvaj G, Orleans M, Beaty B. Oral intake policies on labor and delivery: a national survey. J Clin Anesthesia 1998:10:449-51.

11 Scheepers HCJ, Essed GGM, Brouns F. Aspects of food and fluid intake during labor: policies of midwives and obstetricians in the Netherlands. Eur J Obstet Gynecol Reprod Biol 1998;78:37-40.

12 CNM Data Group. Intrapartum nutrition: what happens in midwifery care? J Nurse Midwifery 1999;44:135-8.

13 Yiannouzis $C$. Randomised controlled trial measuring the effects on labour of offering a light, low fat diet. In: Simms C, McHaffie $\mathrm{H}$, Renfrew M, Ashurst H, eds. The Midwifery Research Database (MIRIAD): a sourcebook of information about research in midwifery. Cheshire: Books for Midwives Press, 1994:196-7.

14 Scrutton MJL, Metcalfe GA, Lowy C, Seed PT, O’Sullivan G. Eating in labour. Anaesthesia 1999;54:329-34.

15 Kubli M, Scrutton MJ, Seed PT, O’Sullivan G. An evaluation of isotonic 'sport drinks' during labor. Anesth Analg 2002;94:404-8.

16 Scheepers HC), Thans MCJ, de Jong PA, Essed GG, Le Cessie S, Kanhai HH. A double-blinded randomised, placebo controlled study on the influence of carbohydrate solution intake during labor. $\mathrm{Br}$ J Obstet Gynecol 2002;109:178-81.

17 Tranmer JE, Hodnett ED, Hannah ME, Stevens BJ. The effect of unrestricted oral carbohydrate intake on labor progress. J Obstet Gynecol Neonatal Nurs 2005;34:319-28.

18 Parsons M. A midwifery practice dichotomy on oral intake in labor. Midwifery 2004;20:72-81.

19 Lewis P. Not to our taste: midwives failure to feed women in labor. Pract Midwife 1998;1:4-5.

20 Sleutel M, Golden SS. Fasting in labor: relic or requirement. J Obste Gynecol Neonatal Nurs 1999;28:507-12.

21 O'Sullivan G, Scrutton M. NPO during labor: is there any scientific validation? Anesthesiol Clin North America 2003;21:87-98.

22 Watanabe T, Minakami H, Sakata Y, Matsubara S, Tamura N, Obara $\mathrm{H}$, et al. Effect of labor on maternal dehydration, starvation, coagulation, and fibrinolysis. J Perinat Med 2001;29:528-34.

23 Jones B, Jarvis P, Lewis JA, Ebbutt AF. Trials to assess equivalence: the importance of rigorous methods. BMJ 1996;313:36-9.

24 O’Sullivan G, Liu B, Shennan AH. Oral intake during labor. Int Anesthesiol Clin 2007;45:133-47

25 Scheepers HCJ, de Jong PA, Essed GGM, Kanhai HHH. Carbohydrate solution intake during labor just before the start of the second stage: a double-blind study on metabolic effects and clinical outcome. $\mathrm{Br}$ J Obstet Gynecol 2004;111:1382-7.

26 Nimmo WS, Wilson J, Prescott LF. Narcotic analgesics and delayed gastric emptying during labour. Lancet 1975;1:890-3

27 Zimmermann DL, Breen TW, Fick G. Adding fentanyl $0.0002 \%$ to epidural bupivacaine $0.125 \%$ does not delay gastric emptying in labouring patients. Anesth Analg 1996;82:612-6

28 Porter JS, Bonello F, Reynolds F. The influence of epidural administration of fentanyl infusion on gastric emptying in labour. Anaesthesia 1997;52:1151-6

29 Kluger MT, Short TG. Aspiration during anaesthesia: a review of 133 cases from the Australian Anaesthesia Incident Monitoring Study (AIMS). Anaesthesia 1999;54:19-26.

30 Simkin P. Stress, pain and catecholamines in labor, part 2. Stres associated with childbirth events: a pilot survey of new mothers. Birth 1986;13:234-40.

31 Frye A. Nourishing the mother. Midwifery Today 1994;31:25-6.

32 Parsons M, Bidewell J, Griffiths R. A comparative study of the effect of food consumption on labour and birth outcomes in Australia. Midwifery 2007;23:131-8.

33 Kollef $\mathrm{MH}$. The prevention of ventilator-associated pneumonia. $N$ Engl J Med 1999;340:627-34

Accepted: 17 December 2008 